\title{
Double Quantum Dot Floquet Gain Medium
}

\author{
J. Stehlik, ${ }^{1}$ Y.-Y. Liu, ${ }^{1}$ C. Eichler, ${ }^{1}$ T. R. Hartke, ${ }^{1}$ X. Mi, ${ }^{1}$ M. J. Gullans, ${ }^{2}$ J. M. Taylor, ${ }^{2}$ and J. R. Petta ${ }^{1}$ \\ ${ }^{1}$ Department of Physics, Princeton University, Princeton, New Jersey 08544, USA \\ ${ }^{2}$ Joint Quantum Institute, National Institute of Standards and Technology, \\ Gaithersburg, Maryland 20899, USA \\ and Joint Center for Quantum Information and Computer Science, \\ University of Maryland, College Park, Maryland 20742, USA
}

(Received 27 July 2016; published 7 November 2016)

\begin{abstract}
Strongly driving a two-level quantum system with light leads to a ladder of Floquet states separated by the photon energy. Nanoscale quantum devices allow the interplay of confined electrons, phonons, and photons to be studied under strong driving conditions. Here, we show that a single electron in a periodically driven double quantum dot functions as a "Floquet gain medium," where population imbalances in the double quantum dot Floquet quasienergy levels lead to an intricate pattern of gain and loss features in the cavity response. We further measure a large intracavity photon number $n_{c}$ in the absence of a cavity drive field, due to equilibration in the Floquet picture. Our device operates in the absence of a dc current-one and the same electron is repeatedly driven to the excited state to generate population inversion. These results pave the way to future studies of nonclassical light and thermalization of driven quantum systems.

DOI: 10.1103/PhysRevX.6.041027

Subject Areas: Condensed Matter Physics,

Quantum Physics,

Semiconductor Physics
\end{abstract}

\section{INTRODUCTION}

A qubit coupled to a microwave resonator allows the study of fundamental light-matter interactions at the level of single photons [1]. The circuit quantum electrodynamics (cQED) architecture enables the exploration of a variety of phenomena. Measurements of the transmission through a superconducting cavity have been used to read out both superconducting [2] and spin qubits [3]. Qubits separated by relatively large distances have been coherently coupled using a cavity bus [4,5]. Finally, cQED enables the generation of classical and nonclassical light [6-9].

Light sources based on the cQED architecture generally use two different approaches. The first approach relies on coherently transferring an excitation from a qubit to a cavity. Early work demonstrated single photon generation by applying a $\pi$ pulse to a qubit to drive a transition from the ground state to the excited state. The qubit was then brought into resonance with the cavity for a short period of time, thereby transferring the excitation from the qubit to the cavity [6]. $n$-photon Fock states were generated by repeating this process many times [9]. A second approach utilizes a source-drain bias to drive a current through a Cooper pair box or a double quantum dot (DQD) [10,11]. Here, the source-drain bias continually repumps the excited

Published by the American Physical Society under the terms of the Creative Commons Attribution 3.0 License. Further distribution of this work must maintain attribution to the author(s) and the published article's title, journal citation, and DOI. state of the artificial atom, leading to population inversion and microwave frequency photoemission. For sufficiently high pumping rates, a transition to above-threshold masing has been achieved $[7,8]$.

When qubits are strongly driven, intriguing quantum effects emerge, including Landau-Zener-Stückelberg interference [12,13], lasing without inversion [14], and vacuum squeezing $[15,16]$. The combination of superconducting resonators and semiconductors-in the form of DQDsprovides a versatile, electrically tunable quantum system that interacts strongly with light $[8,17,18]$.

In this paper, we show that a periodically driven single electron confined to a cavity-coupled DQD functions as a "Floquet gain medium," where population imbalances in the DQD Floquet quasienergy levels lead to an intricate pattern of gain and loss features in the cavity response $[12,13,19]$. The operating regime of the device is distinct from previous work on voltage-biased Josephson junctions and semiconductor DQDs, where a relatively large current flow was required to achieve photoemission [11]. Instead, our device is operated in Coulomb blockade, such that the net current flow through the device is negligible. One and the same electron is repeatedly driven to the excited state to generate population inversion. The drive field modifies the effective qubit level diagram and leads to the formation of a ladder of Floquet bands, as shown in Fig. 1(c) [20]. Periodic driving of the level detuning results in an effective population inversion that depends sensitively on the drive parameters and band alignment. An intricate photon emission pattern emerges as a result of cavity coupling, periodic 
driving, and strong electron-phonon coupling [21]. Our results can be understood in analogy to lasing without inversion in atomic physics [14].

\section{DRIVEN DQD GAIN MEDIUM}

Our experimental setup is illustrated in Figs. 1(a) and 1 (b) and consists of a half-wavelength $(\lambda / 2)$ microwave cavity that contains a DQD. The cavity has a center frequency $\omega_{c} / 2 \pi=7.553 \mathrm{GHz}$ and linewidth $\kappa / 2 \pi=$ $1.5 \mathrm{MHz}$, resulting in a quality factor $Q \approx 5000$. A single InAs nanowire DQD is placed at an antinode of the cavity electric field [see SEM image in Fig. 1(d)] [3]. The DQD confinement potential is generated by five gate electrodes that are located beneath the nanowire [22]. The left $V_{L}$ and right $V_{R}$ gate voltages are used to tune the chemical potential of the left dot $\mu_{L}$ and right dot $\mu_{R}$, thereby setting the interdot detuning $\epsilon(t)=\mu_{R}-\mu_{L}$. Measurements are taken near an interdot charge transition, where a single excess electron occupies either the left or right quantum dot.

To enhance the electric dipole coupling of this electron to the cavity electric field, the nanowire source contact is connected to the cavity center pin, resulting in a chargecavity coupling rate $g_{0} / 2 \pi \approx 80 \mathrm{MHz}$. We probe the cavity by weakly driving the input port of the cavity at frequency $\omega_{\text {in }} / 2 \pi$ and power $P_{\text {in }} \approx-120 \mathrm{dBm}$; see Fig. 1(a). We detect the radiation emitted from the cavity using a multistage amplification process starting with a nearly quantum-limited Josephson parametric amplifier

(a)
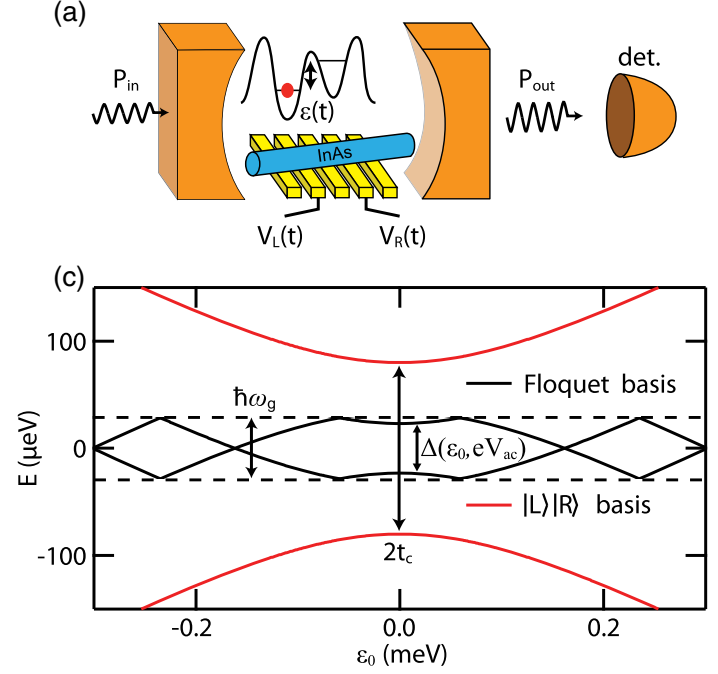

(see Appendix A for a detailed description of the experimental setup) [23-25].

The InAs nanowire DQD is described by a charge qubit Hamiltonian [26,27]:

$$
H_{\mathrm{DQD}}=\frac{\epsilon(t)}{2} \sigma_{z}+t_{c} \sigma_{x} .
$$

Here, $\sigma_{x}$ and $\sigma_{z}$ are the Pauli operators in the left-right charge basis and $t_{c}=80 \pm 10 \mu \mathrm{eV}$ is the interdot tunnel coupling. For $\epsilon_{0}<0$, the excess electron resides in the right dot (denoted $|R\rangle$ ) in the absence of any microwave excitation. The DQD is periodically driven at frequency $\omega_{g} / 2 \pi$ by applying oscillating voltages $V_{L}(t)$ and $V_{R}(t)$ to the gates. The relative amplitude and phase of these voltages are set such that [28]

$$
\epsilon(t)=\epsilon_{0}+e V_{\mathrm{ac}} \sin \left(\omega_{g} t\right) .
$$

Here, $e$ is the electron charge, $V_{\text {ac }}$ is the amplitude of the microwave drive, and $\epsilon_{0}$ is the offset detuning (see Appendices A 1 and A 2 for details). To prevent occupation of the cavity with thermal photons, the experiments are performed in a dilution refrigerator with a base temperature $T \sim 20 \mathrm{mK}$.

In this work, we focus on the strong driving limit of the DQD defined by $e V_{\mathrm{ac}} / \hbar \geq \omega_{g}$ and investigate the effects of this driving on the microwave field in the cavity. As mentioned in the Introduction, such a strongly driven twolevel system coupled to a cavity is analogous to systems
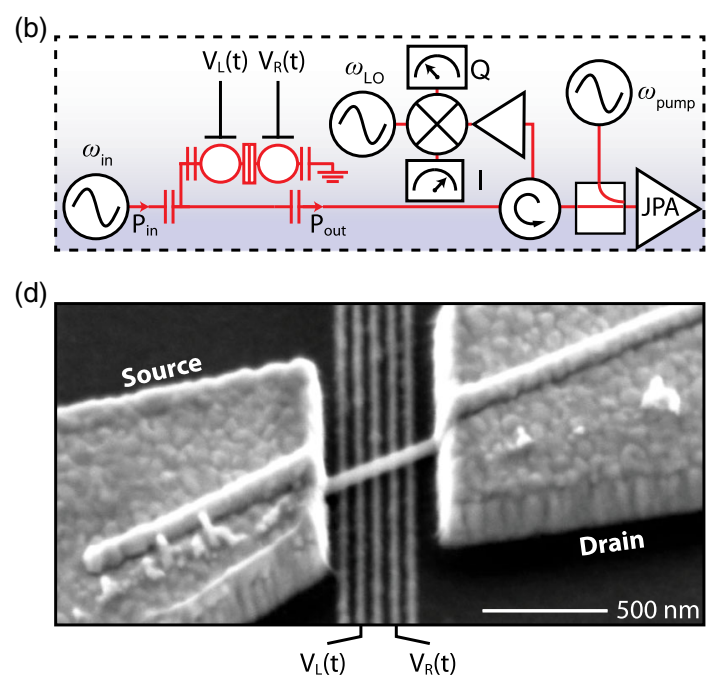

FIG. 1. (a) Driven double quantum dot photon source. The gain medium consists of a DQD whose energy level detuning $\epsilon(t)$ is driven periodically. A cavity containing the DQD is probed using a weak input signal with power $P_{\text {in. }}$. The signal exiting the cavity with power $P_{\text {out }}$ is incident on a detector. (b) Circuit diagram illustrating the DQD, cavity, and linear amplification chain. (c) DQD energy level diagram, with the bonding and antibonding charge states shown in red. Interdot tunnel coupling results in a level splitting of $2 t_{c}$ at $\epsilon_{0}=0$. In the Floquet basis the energy level spectrum is defined only modulo $\hbar \omega_{g}$, resulting in folding of the DQD levels. The splitting of the folded levels is $\Delta\left(\epsilon_{0}, e V_{\mathrm{ac}}\right)$. (d) Scanning electron microscope image of the sample. A single InAs nanowire is placed across five bottom gates and electrically contacted by source and drain electrodes. The detuning parameter is driven by applying oscillating voltages $V_{L}(t)$ and $V_{R}(t)$ to two of the gates. 
commonly studied in atomic physics to realize lasing without inversion [14]. An important distinction, however, is that, because of the strong driving, we are not allowed to make the rotating wave approximation in describing the two-level dynamics. Instead, we use Floquet analysis to derive an effective model for the dynamics of the Floquet quasienergy states [shown in Fig. 1(c) for zero drive amplitude] $[13,20]$. This approach is crucially needed in this strongly driven limit because it allows us to treat the driving field in a nonperturbative manner. In analyzing this problem theoretically, we find a complex interference between drive-assisted photon emission and absorption arising from these nonperturbative drive processes. The importance of these effects on the resulting cavity dynamics leads us to describe this DQD cavity-coupled system as a Floquet gain medium.

\section{CAVITY GAIN}

We measure the cavity power gain $G$ in the presence of the periodic drive. The power gain is defined as $G=C P_{\text {out }} / P_{\text {in }}$, where $P_{\text {out }}$ is the cavity output power and $C$ is a normalization constant set such that $G=1$ with the device configured in Coulomb blockade [8,11]. In Fig. 2(a), we plot $G$ as a function of $e V_{\text {ac }}$ and $\epsilon_{0}$ for $\omega_{g} / 2 \pi=13.75 \mathrm{GHz}$. The cavity gain data exhibit a distinctive interference pattern, with oscillations between regions of cavity gain $(G>1)$ and cavity loss $(G<1)$, as $e V_{\mathrm{ac}}$ and $\epsilon_{0}$ are varied. The boundary of this interference pattern has a " $\mathrm{V}$ " shape that traces out the region where $\left|\epsilon_{0}\right| \sim e V_{\mathrm{ac}}$. Inside this region the ac drive sweeps the level detuning through $\epsilon=0$ and the gain displays an interference pattern [7]. Outside this region the transmission through the cavity is decoupled from the DQD dynamics and $G=1$.

With $\epsilon_{0}=0$, the interference pattern shown in Fig. 2(a) exhibits striking oscillations in $G$ as a function of $e V_{\text {ac }}$. To further investigate these oscillations, we measure the cavity

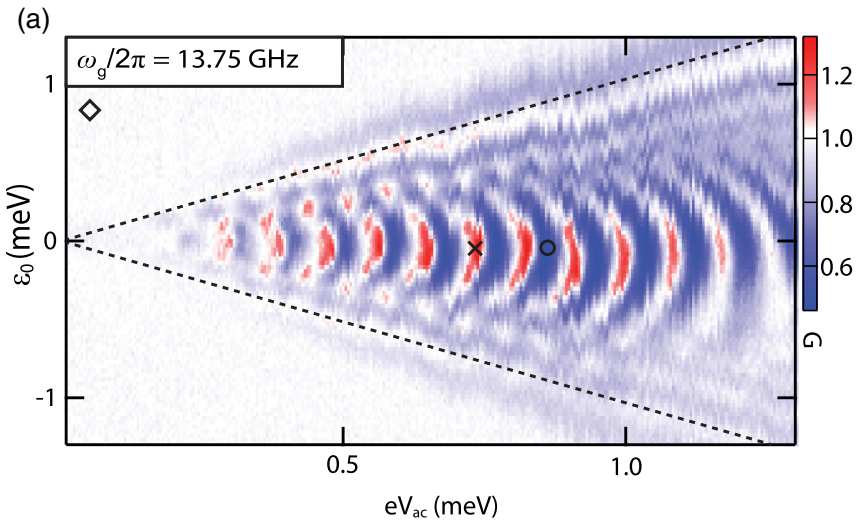

gain as a function of input frequency $\omega_{\text {in }} / 2 \pi$ [Fig. 2(b)] at locations in the interference pattern where there is gain and loss. The conditions $\epsilon_{0}=0$ and $e V_{\mathrm{ac}}=0.73 \mathrm{meV}$ correspond to a location where $G=1.2$, indicating that the Floquet gain medium is effectively inverted. Here, the cavity gain is well fit by a Lorentzian with a FWHM of $1.2 \mathrm{MHz}$, significantly narrower than the bare cavity linewidth $\kappa / 2 \pi=1.5 \mathrm{MHz}$. Alternatively, when we configure the driving parameters to a region of microwave loss $\left(\epsilon_{0}=0, e V_{\mathrm{ac}}=0.85 \mathrm{meV}\right)$, the cavity gain is noticeably broader with a FWHM $=2.1 \mathrm{MHz}$ and peak gain of 0.49 , indicating additional loading of the cavity. Here, the Floquet gain medium is not inverted. In comparison, the green curve in Fig. 2(b) shows the cavity gain in the region outside of the interference pattern (where $\left|\epsilon_{0}\right| \gg e V_{\mathrm{ac}}$ ). In this region, the FWHM $=1.5 \mathrm{MHz}$ is equal to the bare cavity linewidth, indicating that the cavity is not being affected by the charge dynamics of the DQD.

We note that the maximum gain achieved here, $G \sim 1.2$, is quite modest, but comparable to gains achieved in experiments involving driven superconducting qubits $[29,30]$. The gain could be increased by improving the cavity quality factor, increasing $g_{0}$, and reducing the amount of charge noise in the device. Much higher power gains can be achieved by operating the DQD at finite bias [8]. However, as noted in Ref. [19], periodic driving may allow for more control over the statistics of the emitted field.

\section{PHOTON EMISSION IN THE ABSENCE OF A CURRENT}

We now show that the strongly driven DQD emits light into the cavity mode in the absence of an input field. The intracavity photon number $n_{c}$ is plotted as a function of $\epsilon_{0}$ and $e V_{\text {ac }}$ in Fig. 3(a). These data are obtained by measuring the cavity output power in a $2.5 \mathrm{MHz}$ band around the cavity center frequency (see Appendix A 3). The data

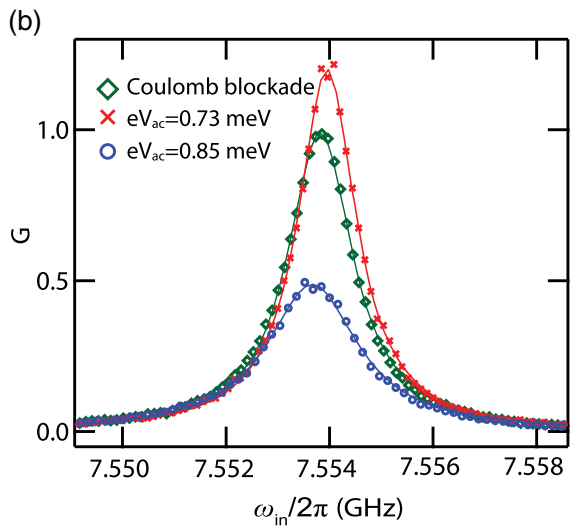

FIG. 2. (a) Floquet gain medium interference pattern. The cavity power gain $G$ plotted as a function of offset detuning $\epsilon_{0}$ and ac drive amplitude $e V_{\text {ac }}$. A pattern of alternating gain $(G>1$, red regions) and loss ( $G<1$, blue regions) is observed. (b) $G$ plotted as a function of $\omega_{\text {in }} / 2 \pi$ with the DQD in Coulomb blockade (green diamond), with the DQD drive configured for loss $\left(e V_{\mathrm{ac}}=0.85 \mathrm{meV}\right.$, blue circle), and with the DQD drive configured for gain $\left(e V_{\mathrm{ac}}=0.73 \mathrm{meV}\right.$, red cross). Solid lines are Lorentzian fits to the data. 


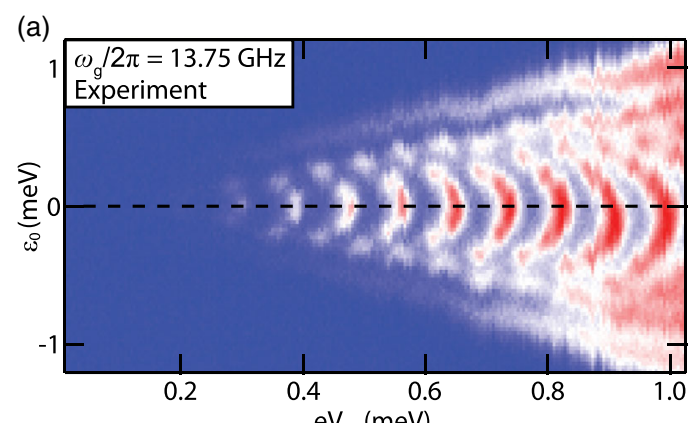

(c)

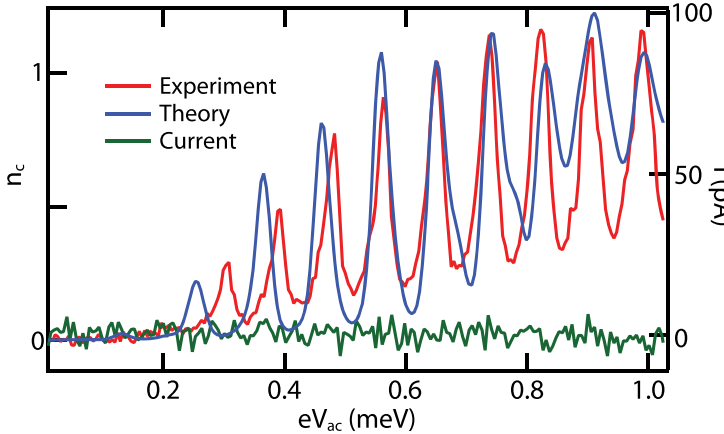

(b)

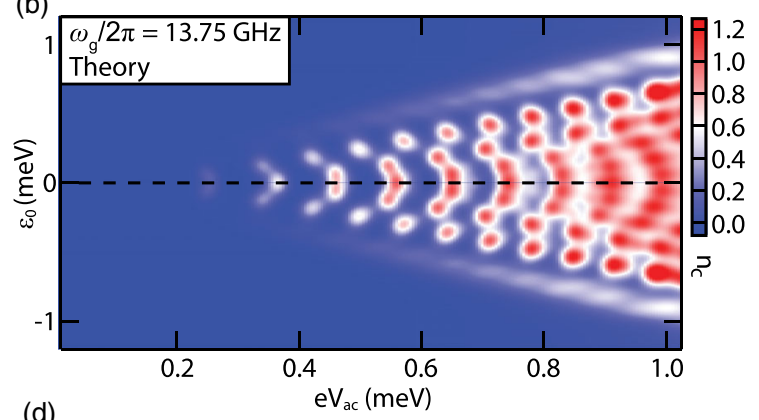

(d)

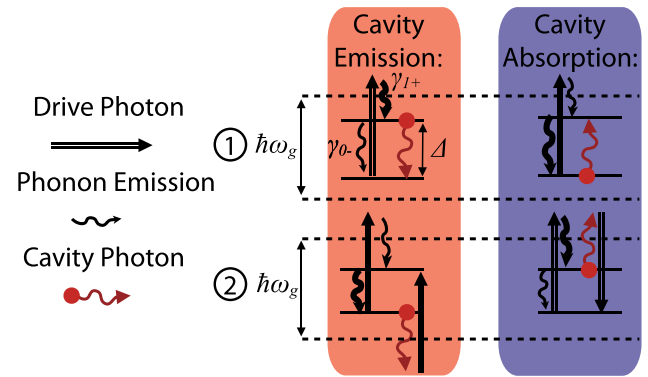

FIG. 3. (a) Photon number interference. $n_{c}$ measured as a function of $\epsilon_{0}$ and $e V_{\text {ac }}$ with no cavity drive. (b) Calculated $n_{c}$ as a function of $\epsilon_{0}$ and $e V_{\text {ac }}$, showing good qualitative agreement with the data. Panels (a) and (b) are plotted using the same color scale. (c) Measured and calculated $n_{c}$ as a function of $e V_{\mathrm{ac}}$ for $\epsilon_{0}=0$. Overlaid is the measured current $I$ through the DQD. Notice that while photons are being emitted, no dc current is detected. (d) Energy level diagrams for emission and absorption peaks. There are two configurations in which population inversion and photon emission occur. In the first case [labeled as (1)], the quasienergy splitting $\Delta=\hbar \omega_{\mathrm{c}}$ and the DQD can directly emit a cavity photon. In the second case [labeled as (2)], $\Delta=\hbar \omega_{\mathrm{g}}-\hbar \omega_{\mathrm{c}}$ and the DQD can simultaneously absorb a drive photon and emit a cavity photon. The equilibrium population is determined by a competition between drive-assisted phonon emission that relaxes or excites the DQD in the Floquet basis at the rates $\gamma_{n-}$ and $\gamma_{n+}$, respectively. Here $n$ denotes the number of energy quanta absorbed from the drive (see discussion surrounding Eq. 6). The schematic illustrates the processes $\gamma_{0-}$ and $\gamma_{1+}$. Dominant phonon relaxation processes are indicated by the bolder arrows in the schematic.

feature oscillations in $n_{c}$ with a periodicity of approximately $100 \mu \mathrm{eV}$ in the drive amplitude $e V_{\mathrm{ac}}$. Oscillations as a function of $\epsilon_{0}$ are also evident, with up to seven peaks visible at $e V_{\mathrm{ac}}=0.8 \mathrm{meV}$. The locations of the maxima and minima in $n_{c}$ are aligned with the maxima and minima observed in the cavity power gain $G$ [see Fig. 2(a)]. We note that Fig. 3(a) features a slowly rising background, which we attribute to heating due to the strong microwave drive (see Appendix C).

While the device emits photons in a wide range of parameter space, there is no detectable current flow through the device [see Fig. 3(c)]. Following previous work, current measurements are performed using a current preamplifier that is connected to the voltage node of the resonator [8]. A detailed circuit diagram is shown in Fig. 4. The lack of current supports the two-level approximation made in defining the DQD Hamiltonian. It also suggests that the emission can be interpreted as resulting from a single electron being repeatedly forced into the excited state only to decay by producing a cavity photon. Such processes are often referred to as Sisyphus pumping [31], as this is analogous to Sisyphus, the character in Greek mythology who eternally pushes a boulder up a hill only for it to roll back down (relax). The experiments presented here are in contrast with previous quantum dot photon emission studies, where a source-drain bias was applied across a DQD to induce an inelastic current flow [11,32,33].

\section{CHARGE-CAVITY COUPLING IN THE PRESENCE OF PHONONS}

The intracavity photon number $n_{c}$ displays a pattern that is suggestive of Landau-Zener-Stückleberg interference $[12,34]$. However, it is not possible to achieve population inversion in a driven two-level system without including some modification of the dissipation by the drive, as seen in lasing without inversion [14] or in Raman phonon emission [35]. A qualitative picture of drive-assisted thermalization leading to inversion is, nevertheless, possible. In Fig. 3(d), we show energy level diagrams for specific driving conditions that lead to population inversion and large intracavity photon numbers. The inversion, where Floquet quasienergy eigenstates provide gain for the cavity, arises from a competition between bare phonon relaxation and drive-assisted phonon relaxation, where the system both emits a phonon and absorbs a drive photon. 


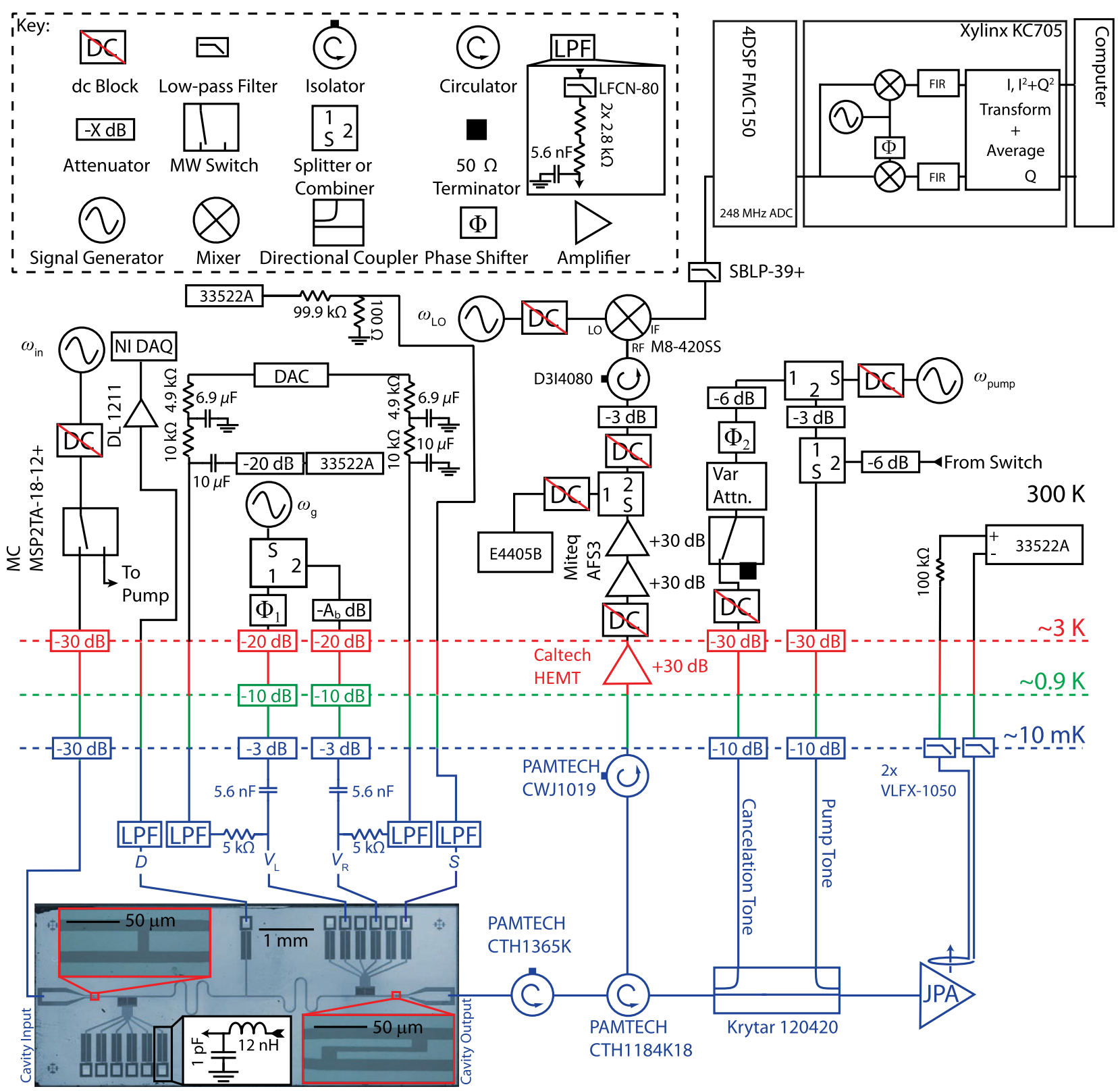

FIG. 4. Complete circuit diagram of the experimental setup. We probe the cavity transmission using a tone at frequency $\omega_{\text {in }}$. The cavity output field is amplified using a JPA. The JPA is biased using a small magnetic coil fed from an Agilent 33522A function generator. The pump field at frequency $\omega_{\text {pump }}$ is supplied using a directional coupler. At room temperature the pump field is also split and phase shifted to provide the cancellation field, which is optionally used to remove the pump field that is reflected off the JPA. The resulting signal is further amplified, before being sent either to an Agilent E4405B spectrum analyzer or downconverted to $20.48 \mathrm{MHz}$ and digitized using a 4DSP FMC150 board. A final digital downconversion and digital filtering stage then recovers the signal. To generate the Floquet gain medium time varying gate voltages are applied. The dc part is generated using a digital to analog converter (DAC) that is heavily filtered both at room temperature and in the $10 \mathrm{mK}$ stage. The ac excitation at frequency $\omega_{\mathrm{g}}$ is provided using a signal generator and added to the dc part via a bias-tee circuit. The current is measured using a DL 1211 preamplifier and digitized using a NI DAQ.

To describe this process more quantitatively, we explicitly account for the electron-phonon interaction by adding to Eq. (1) the following term:

$$
H_{\mathrm{ep}}=\sum_{k \nu} \hbar \omega_{k \nu} a_{k \nu}^{\dagger} a_{k \nu}+\lambda_{k \nu} \sigma_{z}\left(a_{k \nu}+a_{k \nu}^{\dagger}\right)
$$

where $k$ refers to the phonon wave vector along the nanowire, $\nu$ indexes the transverse phonon modes, $\omega_{k \nu}$ is the phonon dispersion, $a_{k \nu}\left(a_{k \nu}^{\dagger}\right)$ is the bosonic annihilation (creation) operator for the phonons, and $\lambda_{k \nu}$ is the electronphonon coupling constant. The total Hamiltonian maps to the spin-boson model [36], with the caveat that one of the 
bosonic modes is the cavity field. In the absence of the drive $\left(e V_{\mathrm{ac}}=0\right)$, the spontaneous phonon relaxation between the two eigenstates of Eq. (1) is

$$
\begin{gathered}
\gamma\left(\omega_{d}\right)=2 \pi \cos ^{2} \theta \mathcal{J}\left(\omega_{d}\right), \\
\mathcal{J}(\omega)=\sum_{k \nu}\left|\lambda_{k \nu}\right|^{2} \delta\left(\omega-\omega_{k \nu}\right),
\end{gathered}
$$

where $\omega_{d}=\sqrt{\epsilon_{0}^{2}+4 t_{c}^{2}}$ is the DQD energy splitting, $\theta=\tan ^{-1}\left(2 t_{c} / \epsilon_{0}\right)$ is the mixing angle between the leftright charge states, and $\mathcal{J}(\omega)$ is the phonon spectral density. The spontaneous emission is accompanied by thermal emission and absorption of the phonons at the rates $\gamma_{\text {th }}\left(\omega_{d}\right)=\gamma\left(\omega_{d}\right) n_{p}\left(\omega_{d}\right)$, where $n_{p}(\omega)=\left(e^{\hbar \omega / k_{B} T}-1\right)^{-1}$ is the Bose-Einstein distribution function at temperature $T$. In this case, the phonons primarily serve to thermalize the DQD charge states with the lattice.

In the presence of the drive $\left(e V_{\mathrm{ac}} \neq 0\right)$, this picture is strongly modified because of the drive-assisted relaxation processes shown in Fig. 3(d). In this case, for every positive integer $n$, we need to account for all phonon emission and absorption processes that absorb $n$ photons from the drive. Each of these processes interacts with phonons at a different frequency, which are characterized by the spectral density [19]

$$
\begin{aligned}
\mathcal{J}_{n \pm}(\Delta) & =\sum_{k \nu}\left|\lambda_{k \nu}\right|^{2}\left|u_{n}\right|^{2} \delta\left(n \omega_{g} \mp \Delta-\omega_{k \nu}\right) \\
& =\left|u_{n}\right|^{2} \mathcal{J}\left(n \omega_{g} \mp \Delta\right),
\end{aligned}
$$

where \pm refers to phonon emission processes where the DQD ends in the upper (lower) Floquet state and $u_{n}$ (found numerically) accounts for the change of basis to the Floquet eigenstates. Each of these spectral densities is associated with a spontaneous phonon emission rate $\gamma_{n \pm}(\Delta)=$ $2 \pi \mathcal{J}_{n \pm}(\Delta)$ and thermal emission and absorption rates $\gamma_{n \pm}(\Delta) n_{p}(n \omega \pm \Delta)$. Figure 3(d) diagrammatically illustrates the two dominant processes at low drive amplitudes: $\gamma_{0-}$ and $\gamma_{1+}$. The first process $\gamma_{0-}$ corresponds to the bare phonon relaxation at the Floquet quasienergy gap $\Delta$. The second process $\gamma_{1+}$, on the other hand, describes a process where the DQD absorbs one quanta from the drive and emits a phonon at the energy $\hbar \omega_{g}-\Delta$, which is greater than zero by the definition of $\Delta$. We see from Eq. (6) that these phonon emission rates are sensitive to the frequency dependence of $\mathcal{J}(\omega)$ and the overlap factor $u_{n}$, the latter of which is a function of the qubit and drive parameters. This should be contrasted with the standard inclusion of dephasing and decay in the Landau-Zener-Stückleberg theory. In this treatment, the decay rates are assumed to be given by their values at $e V_{\text {ac }}=0$ and never lead to population inversion [12]. Our model, on the other hand, allows for the modification of the spectral density by the drive. As a result, the dominant relaxation process, which determines whether there is population inversion or not, is modulated by the system parameters, leading to the oscillating regions of gain and loss observed in Fig. 2(a).

With this understanding, we apply the detailed theory of Ref. [19] to make a direct comparison with the experimental data. Quasistatic charge noise and amplitude variations are included in the model by smoothing the calculated cavity response with a Gaussian of width $50 \mu \mathrm{eV}$ along the $\epsilon_{0}$ axis and $10 \mu \mathrm{eV}$ along the $e V_{\text {ac }}$ axis. A best fit is obtained with $g_{0} / 2 \pi=75 \mathrm{MHz}$ and $t_{c}=85 \mu \mathrm{eV}$, both of which are consistent with our measured values [3]. The calculated $n_{c}$ as a function of $\epsilon_{0}$ and $e V_{\text {ac }}$ is shown in Fig. 3(b) and is in very good agreement with the measured response. We further compare the theoretical predictions with the experimental results in Fig. 3(c), where we plot $n_{c}$ as a function of $e V_{\mathrm{ac}}$ for $\epsilon_{0}=0$. Both measured and calculated responses feature strong oscillations with nearly equivalent periodicities. The discrepancy between the measured and calculated response, especially at high drive amplitudes, may be due to imperfect treatment of the phonon spectrum.

\section{CONCLUSIONS AND OUTLOOK}

In conclusion, we demonstrate that a strongly driven semiconductor DQD emits microwave frequency photons, even in the absence of a dc current. Electron-phonon coupling and the periodic detuning drive result in effective population inversion of the Floquet states. The population inversion is responsible for the cavity power gain and photon emission that are observed in our experiments.

Looking beyond the intriguing interference patterns that are observed, the statistics of the light generated by the driven DQD is predicted to be a sensitive function of $\omega_{g}$ and $e V_{\text {ac }}$ [19]. This means that a single system could span the spectrum of incoherent thermal emission, coherent emision (lasing), and even antibunched light by simply changing the drive parameters. Such tunability is highly desirable and could be used for a detailed study of the transition between classical and nonclassical light. As applied to single photon sources, theory suggests that specific driving parameters will result in $n_{c} \sim 1$ and $g^{(2)}(0)<0.5$ [19].

Our experimental platform may also be useful for spectroscopy. The Floquet energy imbalances that lead to gain and photon emission are very sensitive probes of the spectral properties of the relaxation mechanism of the qubit. In our case, this is the phonon spectral density. The fine features of the gain plot could, therefore, be used to further study lifetime-limiting mechanisms in InAs and other material systems.

As the technology to scale up solid-state qubit systems continues to develop, it becomes possible to imagine hybrid systems composed of multiple classes of mesoscopic quantum devices on the same chip. This work illustrates the 
utility of gate-defined quantum dots in such hybrid mesoscopic systems. Their broad tunability and unique lightmatter interactions, arising from strong electron-phonon coupling, may provide device functionality that is not available in other solid-state qubit systems.

\section{ACKNOWLEDGMENTS}

We thank Bert Harrop for help with sample preparation. Work was supported by the Gordon and Betty Moore Foundation's EPiQS Initiative through Grant No. GBMF4535, with partial support from the National Science Foundation (DMR-1409556 and DMR-1420541). Devices were fabricated in the Princeton University Quantum Device Nanofabrication Laboratory.

\section{APPENDIX A: EXPERIMENTAL METHODS}

\section{Cavity-coupled DQD}

The device consists of a superconducting cavity that contains a single InAs nanowire DQD. An optical micrograph of the sample is shown in the lower lefthand corner of Fig. 4. The $50 \mathrm{~nm}$ diameter nanowire is placed on top of five predefined $\mathrm{Au}$ gate electrodes [37-39]. The gate electrodes are electrically isolated from the nanowire by a $20 \mathrm{~nm}$ layer of silicon nitride $\mathrm{SiN}_{x}$. Source (S) and drain (D) contacts consisting of $20 \mathrm{~nm} \mathrm{Ti} / 180 \mathrm{~nm} \mathrm{Au}$ are defined using electron beam lithography [40]. As in previous experiments, the source contact is directly connected to the center pin of the microwave cavity [3]. Current through the nanowire is then measured using a DL Instruments 1211 current preamplifier, whose output is digitized using a National Instruments (NI) DAQ card. A sourcedrain bias can optionally be applied via an Agilent 33522A function generator.

The microwave cavity consists of a half-wavelength $(\lambda / 2)$ superconducting resonator, which is fabricated by selectively etching a $50 \mathrm{~nm} \mathrm{Nb}$ film that is sputter deposited on an oxidized $\mathrm{Si}$ substrate $(250 \mathrm{~nm})$ with resistivity $\rho>10 \mathrm{k} \Omega \mathrm{cm}$. The cavity center frequency is $f_{c}=\omega_{c} / 2 \pi=7.553 \mathrm{GHz}$ and the cavity linewidth is $\kappa / 2 \pi=1.5 \mathrm{MHz}$, giving a quality factor $Q \approx 5000$.

To increase the photon collection efficiency, we use asymmetric coupling capacitors with $\kappa_{\text {in }} / 2 \pi=0.04 \mathrm{MHz}$ and $\kappa_{\text {out }} / 2 \pi=0.8 \mathrm{MHz}$. Microwave losses are reduced by adding lumped element capacitor-inductor low-pass filters to each gate line. The inductance $L=12 \mathrm{nH}$ and capacitance $C=1 \mathrm{pF}$ are chosen to give a cutoff frequency around $1.4 \mathrm{GHz}$, well below $f_{c}=7.553 \mathrm{GHz}$. From the measured $Q=\omega_{c} /\left(\kappa_{\text {out }}+\kappa_{\text {in }}+\kappa_{\text {int }}\right)$, we estimate the decay rate due to internal losses to be $\kappa_{\text {int }} / 2 \pi \approx$ $0.6 \mathrm{MHz}$, which is an improvement over previous studies [8].

The charge-cavity coupling strength $g_{0}$ is increased by reducing the separation between the nanowire source and

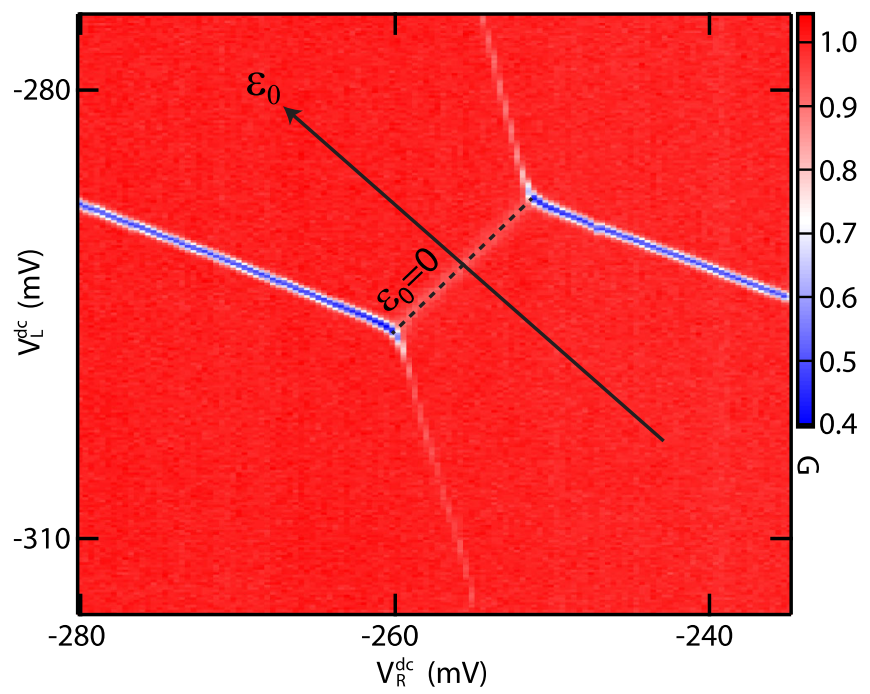

FIG. 5. Cavity power gain $G$ plotted as a function of $V_{R}^{\mathrm{dc}}$ and $V_{L}^{\text {dc }}$, with $e V_{\text {ac }}=0$, showing the charge stability diagram of the DQD. The detuning axis is shown by the solid black arrow. The interdot charge transition, where $\epsilon_{0}=0$, is indicated by the dashed line.

drain contacts to $390 \mathrm{~nm}$. The smaller lithographic dimensions increase the electric field in the region containing the nanowire DQD. As a result, we achieve $g_{0} / 2 \pi \approx 80 \mathrm{MHz}$, which is significantly higher than in previous samples $\left(g_{0} / 2 \pi \approx 30 \mathrm{MHz}\right)[3,8]$.

\section{Detuning drive setup}

The DQD energy level detuning $\epsilon(t)$ is driven by applying time-dependent voltages with frequency $f_{g}=$ $\omega_{g} / 2 \pi$ to two of the gate electrodes $V_{L}(t)$ and $V_{R}(t)$. The microwave voltages are added to the dc voltages $V_{L}^{\mathrm{dc}}$ and $V_{R}^{\mathrm{dc}}$, which are used to generate the DQD confinement potential. These dc voltages are generated at room temperature using heavily filtered DACs. As result, $V_{L}(t)$ and $V_{R}(t)$ become

$$
\begin{gathered}
V_{L}(t)=V_{L}^{\mathrm{dc}}+V_{L}^{\mathrm{ac}} \sin \left(\omega_{g} t\right), \\
V_{R}(t)=V_{R}^{\mathrm{dc}}+V_{R}^{\mathrm{ac}} \sin \left(\omega_{g} t+\phi_{1}\right) .
\end{gathered}
$$

Here, $V_{L}^{\mathrm{ac}}$ and $V_{R}^{\mathrm{ac}}$ are the amplitudes of the microwave signals that are applied to the gates. In general, these amplitudes are different due to imperfections in the two coaxial lines and the lumped-element $L C$ filters that are located on each gate line. The relative phase $\phi_{1}$ is due to different coax cable lengths and a variable phase shifter. A fixed attenuator, $A_{b}=3 \mathrm{~dB}$, is used to balance $V_{R}^{\mathrm{ac}}$ and $V_{L}^{\mathrm{ac}}$. By adjusting the phase shifter such that $\phi_{1} \approx \pi$, we achieve driving that is primarily along the interdot detuning axis shown in Fig. 5. 


\section{Amplification chain and calibration of the intracavity photon number}

To detect the relatively weak photon emission from the cavity, we employ a Josephson parametric amplifier (JPA) $[23,25,41-43]$. The cavity output is first passed through an isolator and a circulator before being amplified by the JPA. The signal is further amplified by a HEMT amplifier with a $4 \mathrm{~K}$ noise temperature and two Miteq room-temperature amplifiers. The JPA is driven by a strong microwave pump tone at frequency $\omega_{\text {pump }} / 2 \pi=7.54776 \mathrm{GHz}$. The pump tone is added to the cavity output signal using a Krytar directional coupler. Because of imperfect isolation, a small portion of the pump tone may reflect off of the JPA and reach the output port of the cavity. The Krytar directional coupler is also used to couple a cancellation tone to the cavity output port. The cancellation tone originates from the same signal generator, but it is attenuated and phase shifted to result in nearly perfect destructive interference of the reflected pump tone. The cancel tone can be turned off via a room-temperature microwave switch.

After being amplified the signal is either measured using an Agilent E4405B spectrum analyzer or down-converted to $20.48 \mathrm{MHz}$ using a Marki M8-420SS mixer (with a local oscillator $\omega_{\mathrm{LO}} / 2 \pi=7.574295 \mathrm{GHz}$ ). The downconverted signal is digitized by a 4DSP FMC150 fast analog-to-digital converter (ADC) board and processed using a Xylinx KC705 field-programmable gate array (FPGA) board. Our FPGA program first generates the $I$ $(Q)$ quadratures by multiplying the signal with a $20.48 \mathrm{MHz}$ sine (cosine) wave. The output is then filtered using a 200 element finite impulse response (FIR) filter with a $2.5 \mathrm{MHz}$ bandwidth. The resulting signal is either kept as $I$ and $Q$ streams and averaged or $I^{2}+Q^{2}$ is calculated and then averaged. For gain measurements, we measure the time-averaged quadratures $\{\bar{I}, \bar{Q}\}$. We can extract the power gain $G=C P_{\text {out }} / P_{\text {in }}$ by noting its relationship with the transmitted amplitude $T$ :

$$
G=C|T|^{2}=C|\bar{I}+i \bar{Q}|^{2}
$$

As in previous experiments, the normalization constant $C$ is defined such that $G=1$ with the device configured in Coulomb blockade [3].

For photon number measurements, we measure $\left\{\overline{I^{2}+Q^{2}}\right\}$, which is equivalent to measuring the power in the FIR-defined band. We note that this power is proportional to the intracavity photon number $n_{c}$, up to some constant noise offset $N_{\text {offset }}$. $N_{\text {offset }}$ is determined by first measuring the power spectral density at the output of the detection chain using an Agilent E4405B spectrum analyzer. When the parametric amplifier is turned off, we measure $S_{\text {off }}(\omega)$. The power that we obtain is proportional to $N_{\text {offset }}$. Here, $N_{\text {offset }}$ refers to noise photon flux per unit bandwidth referenced to the input of the JPA. When the parametric amplifier is turned on, measurements of the
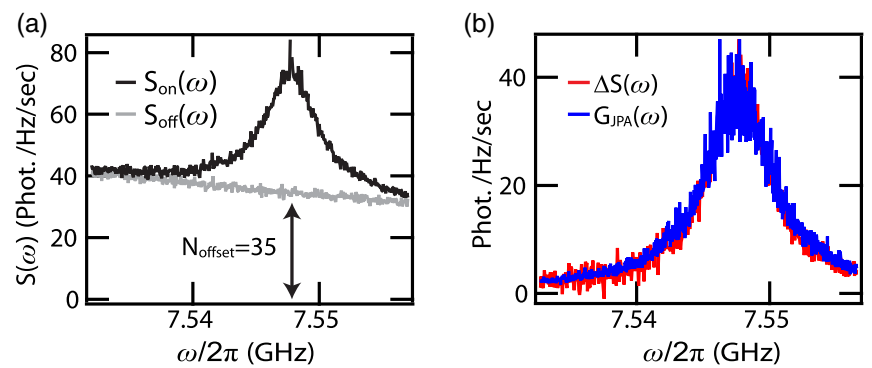

FIG. 6. (a) Measured power spectra $S_{\text {on }}\left(S_{\text {off }}\right)$ taken with the JPA on (off). The peak in the power spectrum acquired with the JPA turned on corresponds to amplified vacuum noise. The noise offset $N_{\text {offset }}$ is referenced to the input of the JPA. (b) JPA gain $G_{\text {JPA }}(\omega)$ overlapped with the difference between the two power spectra, $\Delta S(\omega)=S_{\text {on }}(\omega)-S_{\text {off }}(\omega)$.

power spectral density $S_{\text {on }}(\omega)$ exhibit an additional noise contribution with a Lorentzian shape. We identify the additional noise contribution as amplified vacuum noise. As a result, when referenced back to the input of the JPA, this contribution will be equivalent to a photon flux per unit bandwidth of $G_{\mathrm{JPA}}(\omega)-1$, where $G_{\mathrm{JPA}}(\omega)$ is the JPA gain [44].

We use network analyzer $S$ parameter measurements to independently determine $G_{\mathrm{JPA}}(\omega)$ [see Fig. 6(b)]. We can now scale $\Delta S(\omega)=S_{\text {on }}(\omega)-S_{\text {off }}(\omega)$ to match $G_{\mathrm{JPA}}(\omega)-1$, which fixes the proportionality constant between the absolute measured power and photon flux per unit bandwidth referenced to the input of the JPA. The noise offset in close vicinity to the JPA pump frequency is extracted to be $N_{\text {offset }}=35$ [see Fig. 6(a)] and is attributed to contributions from (i) the HEMT amplifier noise, (ii) losses between the JPA and the HEMT, and (iii) additional noise and losses following the HEMT amplifier.

Knowledge of this noise offset allows us to calibrate the photon flux that arrives at the JPA during cavity emission. We choose the driving conditions $\epsilon_{0}=0$ and $e V_{\text {ac }}=0.82 \mathrm{meV}$ (corresponding to a peak in the cavity emission) to generate a steady photon population $n_{c}$ in the cavity. The total photon flux at the input of the JPA is then $n_{\mathrm{JPA}, \text { in }}=\eta \kappa_{\text {out }} n_{c}$, where $\eta=-3.3 \mathrm{~dB}=0.46$ is the measured transmission coefficient from the cavity to the JPA. We measure the power spectrum $S_{\text {out }}(\omega)$ that results from the cavity emission. Note that for this calibration measurement, we turn off the JPA to avoid the influence of nontrivial filtering effects during the analysis.

Based on this measured power spectrum, we evaluate the intracavity photon number as

$$
n_{c}=\frac{1}{\eta} \frac{\kappa}{\kappa_{\text {out }}} \int \frac{d \omega}{2 \pi} S_{\text {out }}(\omega)=1.1 \pm 0.4 .
$$

Here, the uncertainty is due to (i) uncertainty in the value of $\eta$, which is measured at room temperature, (ii) lithographic errors in the cavity-coupling capacitor 


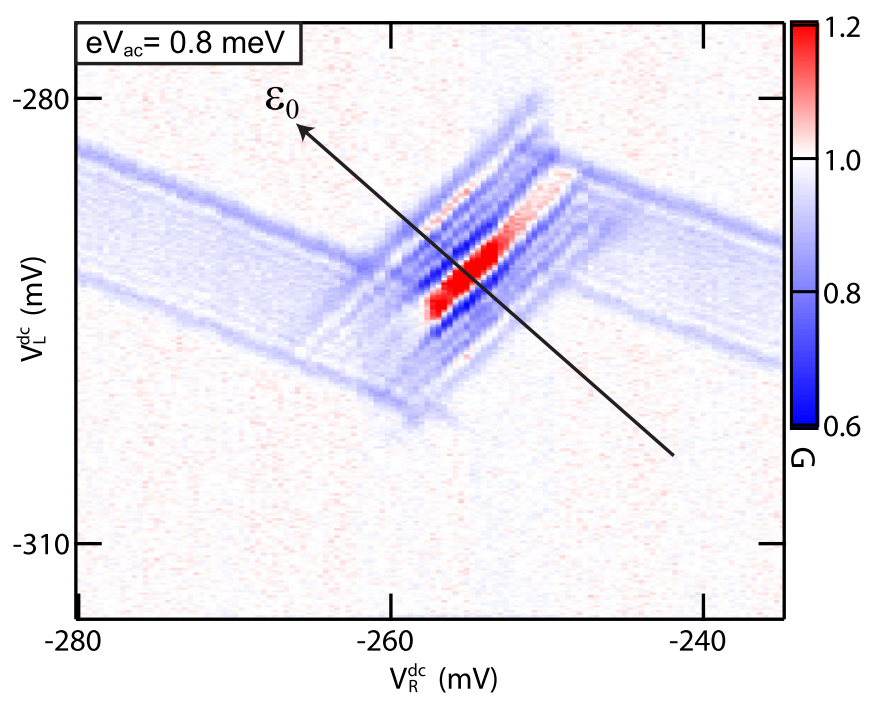

FIG. 7. $G$ plotted as a function of $V_{L}^{\mathrm{dc}}$ and $V_{R}^{\mathrm{dc}}$ for $e V_{\text {ac }}=0.8 \mathrm{meV}$. The plot features strong oscillations parallel to the interdot charge transition line.

dimensions (which influence $\kappa_{\text {in }}$ and $\kappa_{\text {out }}$ ), and (iii) statistical uncertainty in $S_{\text {out }}(\omega)$. We use this knowledge to appropriately scale the photon emission data shown in Fig. 3(a).

\section{APPENDIX B: GAIN VIDEO}

To further explore the gain medium, we plot $G$ as a function of $V_{L}^{\mathrm{dc}}$ and $V_{R}^{\mathrm{dc}}$ for $e V_{\mathrm{ac}}=0.8 \mathrm{meV}$ in Fig. 7. The plot features strong oscillations in $G$. Crucially, the gain and loss fringes are parallel to the interdot detuning line. This signifies that the observed dynamics are independent of the biasing of the DQD energy levels with respect to leads and, thus, strengthens the interpretation that the observed behavior is a purely two-level system phenomenon. The fringes weaken near the triple points of the DQD, which we attribute to photon-assisted tunneling processes with the leads [22]. Our detuning axis purposefully avoids these regions. The evolution of the interference pattern is directly visualized in Movie I of the Supplemental Material [45], where we plot $G$ as a function of $V_{L}^{\mathrm{dc}}$ and $V_{R}^{\mathrm{dc}}$ for increasing $e V_{\mathrm{ac}}$.

\section{APPENDIX C: THEORETICAL MODEL}

To model the periodically driven DQD, we use the Hamiltonian from Eqs. (3) and (4) along with the theoretical methods described in our previous work [19]. To summarize, we first move to a Floquet picture where $H(t)$ is mapped to a time-independent Hamiltonian $H_{F}$ by adding the term $\hbar \omega \hat{N}=\sum_{m} \hbar \omega m|m\rangle\langle m|$, where $m$ is the Floquet index, and converting the functions $e^{-i n \omega t}$ into operators that change the Floquet index by $n[13,20]$. In this Floquet basis, we then integrate out the phonons in a Born-
Markov approximation to derive a master equation describing the DQD dynamics induced by phonon relaxation and absorption. This approach allows us to calculate the steadystate population inversion between the two quasienergy states of the DQD, shown in Fig. 3(d), as well as all multitime DQD correlation functions [e.g., $\left.\left\langle\sigma_{+}(t) \sigma_{-}\left(t^{\prime}\right)\right\rangle\right]$. We then use the steady-state populations and correlation functions to find the photon correlation function $n_{c}$, which is given by the equal-time $\left(t=t^{\prime}\right)$ correlation function of the cavity $\left\langle a^{\dagger}\left(t^{\prime}\right) a(t)\right\rangle$ [19]. All of these calculations are done assuming the phonons are at a temperature $T$, which varies linearly with the drive amplitude due to microwave heating of the sample. More precisely, $T\left(e V_{\text {ac }}\right)=T_{0}+e V_{\text {ac }} \alpha_{T}$, with $T_{0}=0.02 \mathrm{~K}$ the base temperature of the dilution refrigerator, and $\alpha_{T}=$ $0.2(1) \mathrm{K} / \mathrm{meV}$ is chosen to match the oscillation minima in $n_{c}$ [see Fig. 3(c)].

The primary uncertainty in the theoretical model is the precise form for the phonon spectral density $\mathcal{J}(\nu)$. At these energy scales, $J(\nu)$ is dominated by piezoelectric phonons. The separation between the two dots is $d=100 \mathrm{~nm}$, the longitudinal confinement is $a=25 \mathrm{~nm}$ for each dot, the phonon speed of sound in the nanowire and substrate is $c_{n}=4000 \mathrm{~m} / \mathrm{s}$ and $c_{s}=11000 \mathrm{~m} / \mathrm{s}$, respectively, and the DQD relaxation rate at zero detuning (extracted from current measurements) is $\gamma / 2 \pi=0.5 \mathrm{GHz}[46,47]$. At large drive amplitudes, virtual processes involving many quanta of the drive are important, which probe $\mathcal{J}(\nu)$ at high frequencies. As a result, accurate modeling of the data requires a careful treatment of the high-frequency behavior of $\mathcal{J}(\nu)$. We account for this behavior by including, in addition to the coupling to the lowest acoustic branch of the nanowire phonons, coupling of the DQD to acoustic phonons in the substrate $\left(\mathrm{SiN}_{x}\right)$ [47]. This treatment gives rise to the functional form for $\mathcal{J}(\nu)$ :

$$
\begin{aligned}
\mathcal{J}(\nu)= & j_{n} \frac{\sin ^{2}\left(\nu d / 2 c_{n}\right)}{\left(\nu d / 2 c_{n}\right)} e^{-\nu a / c_{n}} \\
& +j_{s} \frac{\nu}{2 c_{s} / d}\left[1-\operatorname{sinc}\left(\nu d / 2 c_{s}\right)\right] e^{-\nu a / c_{s}},
\end{aligned}
$$

where $\operatorname{sinc}(x)=\sin (x) / x$. The parameters $j_{s}$ and $j_{n}$ are determined by the constraint $\gamma=2 \pi \mathcal{J}\left(2 t_{c} / \hbar\right)$, with the ratio $r=j_{s} / j_{n}$ treated as a free parameter when we fit the data in Fig. 3. We find the best fit when $r=6$, with consistent results when $r$ is varied up to $50 \%$ of this value.

[1] A. Blais, R.-S. Huang, A. Wallraff, S. Girvin, and R. Schoelkopf, Cavity Quantum Electrodynamics for Superconducting Electrical Circuits: An Architecture for Quantum Computation, Phys. Rev. A 69, 062320 (2004).

[2] A. Wallraff, D. I. Schuster, A. Blais, L. Frunzio, R.-S. Huang, J. Majer, S. Kumar, S. M. Girvin, and R. J. Schoelkopf, Strong Coupling of a Single Photon to a 
Superconducting Qubit Using Circuit Quantum Electrodynamics, Nature (London) 431, 162 (2004).

[3] K. D. Petersson, L. W. McFaul, M. D. Schroer, M. Jung, J. M. Taylor, A. A. Houck, and J. R. Petta, Circuit Quantum Electrodynamics with a Spin Qubit, Nature (London) 490 , 380 (2012).

[4] M. A. Sillanpaa, J. I. Park, and R. W. Simmonds, Coherent Quantum State Storage and Transfer between Two Phase Qubits via a Resonant Cavity, Nature (London) 449, 438 (2007).

[5] J. Majer, J. M. Chow, J. M. Gambetta, J. Koch, B. R. Johnson, J. A. Schreier, L. Frunzio, D. I. Schuster, A. A. Houck, A. Wallraff, A. Blais, M. H. Devoret, S. M. Girvin, and R. J. Schoelkopf, Coupling Superconducting Qubits via a Cavity Bus, Nature (London) 449, 443 (2007).

[6] A. A. Houck, D. I. Schuster, J. M. Gambetta, J. A. Schreier, B. R. Johnson, J. M. Chow, L. Frunzio, J. Majer, M. H. Devoret, S. M. Girvin, and R. J. Schoelkopf, Generating Single Microwave Photons in a Circuit, Nature (London) 449, 328 (2007).

[7] O. Astafiev, K. Inomata, A. O. Niskanen, T. Yamamoto, Y. A. Pashkin, Y. Nakamura, and J. S. Tsai, Single ArtificialAtom Lasing, Nature (London) 449, 588 (2007).

[8] Y.-Y. Liu, J. Stehlik, C. Eichler, M. J. Gullans, J. M. Taylor, and J. R. Petta, Semiconductor Double Quantum Dot Micromaser, Science 347, 285 (2015).

[9] M. Hofheinz, H. Wang, M. Ansmann, R. C. Bialczak, E. Lucero, M. Neeley, A. D. O'Connell, D. Sank, J. Wenner, J. M. Martinis, and A. N. Cleland, Synthesizing Arbitrary Quantum States in a Superconducting Resonator, Nature (London) 459, 546 (2009).

[10] M. Hofheinz, F. Portier, Q. Baudouin, P. Joyez, D. Vion, P. Bertet, P. Roche, and D. Esteve, Bright Side of the Coulomb Blockade, Phys. Rev. Lett. 106, 217005 (2011).

[11] Y.-Y. Liu, K. D. Petersson, J. Stehlik, J. M. Taylor, and J. R. Petta, Photon Emission from a Cavity-Coupled Double Quantum Dot, Phys. Rev. Lett. 113, 036801 (2014).

[12] S. Shevchenko, S. Ashhab, and F. Nori, Landau-ZenerStückelberg Interferometry, Phys. Rep. 492, 1 (2010).

[13] M. Grifoni and P. Hänggi, Driven Quantum Tunneling, Phys. Rep. 304, 229 (1998).

[14] J. Mompart and R. Corbalán, Lasing Without Inversion, J. Opt. B 2, R7 (2000).

[15] H. J. Kimble, M. Dagenais, and L. Mandel, Photon Antibunching in Resonance Fluorescence, Phys. Rev. Lett. 39, 691 (1977).

[16] C. H. H. Schulte, J. Hansom, A. E. Jones, C. Matthiesen, C. Le Gall, and M. Atature, Quadrature Squeezed Photons from a Two-Level System, Nature (London) 525, 222 (2015).

[17] M. Delbecq, V. Schmitt, F. Parmentier, N. Roch, J. Viennot, G. Fève, B. Huard, C. Mora, A. Cottet, and T. Kontos, Coupling a Quantum Dot, Fermionic Leads, and a Microwave Cavity on a Chip, Phys. Rev. Lett. 107, 256804 (2011).

[18] T. Frey, P. Leek, M. Beck, A. Blais, T. Ihn, K. Ensslin, and A. Wallraff, Dipole Coupling of a Double Quantum Dot to a Microwave Resonator, Phys. Rev. Lett. 108, 046807 (2012).

[19] M. J. Gullans, J. Stehlik, Y.-Y. Liu, C. Eichler, J. R. Petta, and J. M. Taylor, Sisyphus Thermalization of Photons in a Double Quantum Dot, Phys. Rev. Lett. 117, 056801 (2016).
[20] H. Sambe, Steady States and Quasienergies of a QuantumMechanical System in an Oscillating Field, Phys. Rev. A 7, 2203 (1973).

[21] P. Neilinger, S. N. Shevchenko, J. Bogár, M. Rehák, G. Oelsner, D. S. Karpov, O. Astafiev, M. Grajcar, and E. Il'ichev, Landau-Zener-Stückelberg-Majorana Lasing in Circuit QED, Phys. Rev. B 94, 094519 (2016).

[22] W. G. van der Wiel, S. De Franceschi, J. M. Elzerman, T. Fujisawa, S. Tarucha, and L. P. Kouwenhoven, Electron Transport through Double Quantum Dots, Rev. Mod. Phys. 75, 1 (2002).

[23] C. Caves, Quantum Limits on Noise in Linear Amplifiers, Phys. Rev. D 26, 1817 (1982).

[24] B. Yurke, L. R. Corruccini, P. G. Kaminsky, L. W. Rupp, A. D. Smith, A.H. Silver, R.W. Simon, and E. A. Whittaker, Observation of Parametric Amplification and Deamplification in a Josephson Parametric Amplifier, Phys. Rev. A 39, 2519 (1989).

[25] M. A. Castellanos-Beltran, K. D. Irwin, G. C. Hilton, L. R. Vale, and K. W. Lehnert, Amplification and Squeezing of Quantum Noise with a Tunable Josephson Metamaterial, Nat. Phys. 4, 929 (2008).

[26] J. R. Petta, A. C. Johnson, J. M. Taylor, E. A. Laird, A. Yacoby, M. D. Lukin, C. M. Marcus, M. P. Hanson, and A. C. Gossard, Coherent Manipulation of Coupled Electron Spins in Semiconductor Quantum Dots, Science 309, 2180 (2005).

[27] J. Stehlik, Y. Dovzhenko, J. R. Petta, J. R. Johansson, F. Nori, H. Lu, and A. C. Gossard, Landau-Zener-Stückelberg Interferometry of a Single Electron Charge Qubit, Phys. Rev. B 86, 121303 (2012).

[28] B. Roche, R.-P. Riwar, B. Voisin, E. Dupont-Ferrier, R. Wacquez, M. Vinet, M. Sanquer, J. Splettstoesser, and X. Jehl, A Two-Atom Electron Pump, Nat. Commun. 4, 1581 (2013).

[29] G. Oelsner, P. Macha, O. V. Astafiev, E. Il'ichev, M. Grajcar, U. Hübner, B. I. Ivanov, P. Neilinger, and H.-G. Meyer, Dressed-State Amplification by a Single Superconducting Qubit, Phys. Rev. Lett. 110, 053602 (2013).

[30] K. Koshino, H. Terai, K. Inomata, T. Yamamoto, W. Qiu, Z. Wang, and Y. Nakamura, Observation of the Three-State Dressed States in Circuit Quantum Electrodynamics, Phys. Rev. Lett. 110, 263601 (2013).

[31] M. Grajcar, S. H. W. van der Ploeg, A. Izmalkov, E. Il'ichev, H.-G. Meyer, A. Fedorov, A. Shnirman, and G. Schön, Sisyphus Cooling and Amplification by a Superconducting Qubit, Nat. Phys. 4, 612 (2008).

[32] A. Stockklauser, V. F. Maisi, J. Basset, K. Cujia, C. Reichl, W. Wegscheider, T. Ihn, A. Wallraff, and K. Ensslin, Microwave Emission from Hybridized States in a Semiconductor Charge Qubit, Phys. Rev. Lett. 115, 046802 (2015).

[33] J. J. Viennot, M. R. Delbecq, M. C. Dartiailh, A. Cottet, and T. Kontos, Out-of-Equilibrium Charge Dynamics in a Hybrid Circuit Quantum Electrodynamics Architecture, Phys. Rev. B 89, 165404 (2014).

[34] W. D. Oliver, Y. Yu, J. C. Lee, K. K. Berggren, L. S. Levitov, and T.P. Orlando, Mach-Zehnder Interferometry in a Strongly Driven Superconducting Qubit, Science 310, 1653 (2005). 
[35] J. Colless, X. Croot, T. Stace, A. Doherty, S. Barrett, H. Lu, A. Gossard, and D. Reilly, Raman Phonon Emission in a Driven Double Quantum Dot, Nat. Commun. 5, 3716 (2014).

[36] A. J. Leggett, S. Chakravarty, A. T. Dorsey, M. P. A. Fisher, A. Garg, and W. Zwerger, Dynamics of the Dissipative TwoState System, Rev. Mod. Phys. 59, 1 (1987).

[37] C. Fasth, A. Fuhrer, L. Samuelson, V. Golovach, and D. Loss, Direct Measurement of the Spin-Orbit Interaction in a Two-Electron InAs Nanowire Quantum Dot, Phys. Rev. Lett. 98, 266801 (2007).

[38] S. Nadj-Perge, S. M. Frolov, E. P. A. M. Bakkers, and L. P. Kouwenhoven, Spin-Orbit Qubit in a Semiconductor Nanowire, Nature (London) 468, 1084 (2010).

[39] M. D. Schroer and J. R. Petta, Correlating the Nanostructure and Electronic Properties of InAs Nanowires, Nano Lett. 10, 1618 (2010).

[40] D. B. Suyatin, C. Thelander, M. T. Björk, I. Maximov, and L. Samuelson, Sulfur Passivation for Ohmic Contact Formation to InAs Nanowires, Nanotechnology 18, 105307 (2007).

[41] R. Movshovich, B. Yurke, P. G. Kaminsky, A. D. Smith, A. H. Silver, R. W. Simon, and M. V. Schneider, Observation of Zero-Point Noise Squeezing via a JosephsonParametric Amplifier, Phys. Rev. Lett. 65, 1419 (1990).
[42] C. M. Caves, J. Combes, Z. Jiang, and S. Pandey, Quantum Limits on Phase-Preserving Linear Amplifiers, Phys. Rev. A 86, 063802 (2012).

[43] J. Stehlik, Y.-Y. Liu, C. M. Quintana, C. Eichler, T. R. Hartke, and J.R. Petta, Fast Charge Sensing of a Cavity-Coupled Double Quantum Dot Using a Josephson Parametric Amplifier, Phys. Rev. Applied 4, 014018 (2015).

[44] C. Eichler, D. Bozyigit, C. Lang, L. Steffen, J. Fink, and A. Wallraff, Experimental State Tomography of Itinerant Single Microwave Photons, Phys. Rev. Lett. 106, 220503 (2011).

[45] See Supplemental Material at http://link.aps.org/ supplemental/10.1103/PhysRevX.6.041027 for a movie showing the cavity gain as a function of the double quantum dot gate voltages for increasing microwave drive amplitudes.

[46] T. Brandes, Coherent and Collective Quantum Optical Effects in Mesoscopic Systems, Phys. Rep. 408, 315 (2005).

[47] C. Weber, A. Fuhrer, C. Fasth, G. Lindwall, L. Samuelson, and A. Wacker, Probing Confined Phonon Modes by Transport through a Nanowire Double Quantum Dot, Phys. Rev. Lett. 104, 036801 (2010). 